\title{
Review: drug and non-drug treatments are effective in settling problems and night waking in young children but the long term effectiveness of non-drug treatments is more evident
}

Ramchandani $P$, Wiggs $L$, Webb Vet al. A systematic review of treatments for settling problems and night waking in young children. BMJ 2000 Jan 22;320:209-13.

QUESTION: In healthy young children, are drug and non-drug treatments effective for settling problems and night waking?

\section{Data sources}

Studies were identified by searching Medline (1966 to September 1998), EMBASE/Excerpta Medica (1980 to June 1998), PsycLIT (1974 to September 1998), Biological Abstracts (1985 to June 1998), CINAHL (1982 to September 1998), SIGLE (1980 to June 1998), and the Cochrane database (issue 2, 1998); scanning the bibliographies of identified articles and those in reviews and books; handsearching the Journal of Child Psychology and Psychiatry; and by contacting authors and manufacturers of trimeprazine.

\section{Study selection}

Studies were included if they were randomised controlled trials (describing drug or non-drug interventions) of young children ( $\leq 5$ y of age) with an established sleep problem or night waking that had an outcome measure of number of night wakes, time to settle, or number of nights in which these problems occurred.

\section{Data extraction}

Data were extracted on the study design, number and age range of the participants, the referral source, the sleep problem, the intervention and control procedures, the outcome scores and measures, and the assessment times.

\section{Main results}

9 studies met the selection criteria; 4 drug trials, 4 on behavioural treatments, and 1 on a general nondirective educational approach. A meta-analysis was not done because of study heterogeneity. 2 drug trials compared trimeprazine (maximum dose $90 \mathrm{mg}$ ) with placebo. Both showed a statistically significant positive effect of trimeprazine in the short term. Some children treated with trimeprazine, however, continued to wake at night and up to one third did not improve with trimeprazine. Long term results were less favourable. 1 trial compared niaprazine with chlordesmethyl diazepam and found the drugs to be comparable. The fourth drug trial compared trimeprazine as an adjunct to an extinction programme with placebo and found no difference between the groups at end of treatment and at 4 weeks of follow up.

The non-drug interventions investigated were positive routines, graduated extinction, scheduled wakes, extinction or systematic ignoring, modified extinction, educational booklet, and a sleep programme. Scheduled wakenings and extinction reduced night waking compared with the control, a sleep diary, in the short and long term. Positive routines and graduated extinction were also effective in settling problems when compared with waiting list in the short and long term. A non-directive educational booklet showed no benefit over a control procedure. The benefits of a modified extinction procedure and of a booklet giving specific advice were unclear because no untreated control group comparisons were done.

\section{Conclusion}

Both drug and some non-drug treatments are effective in the short term for settling problems and night waking in young children but long term beneficial effects are more evident with non-drug treatments.

\section{COMMENTARY}

Considerable geographic variation exists in the treatment for sleep problems in young children. For example, in a study by Kopferschmitt $e t$ al it was reported that some 12\% of the 11595 children enrolled in kindergarten in the Bas Rhin province in France had been given psychotropic medication for "sleep problems" for more than 6 months and up to 5 years. ${ }^{1}$ In contrast, medication for sleep problems is rarely, if ever, given in the US. There is, however, great interest in the US in the effectiveness of various behavioural approaches for sleep difficulties, which is well documented in a recent review article. ${ }^{2}$

There are 4 lessons we can learn from the present study. Firstly, drugs can have an effect on sleep behaviours for a brief period but there is little evidence that they are helpful in the long term. One reason for this is that sleep problems are often reflections of a specific parental management style ${ }^{3}$ which governs both day and night behaviour of such children. These problems will not be modified by a sleeping pill. Secondly, many parents will not permit their young children to receive psychotropic drugs, hence such studies may use selective and potentially unrepresentative populations. Thirdly, falling asleep and the number of night wakings are influenced by the neurobehavioural development of the infant, especially during the first 6 months. Thus, $75 \%$ of children will not disturb their parents during the night at age 6 months while virtually all do so earlier on. Fourthly, night waking is considered normal in infants. The "caseness" of wakings comes about when children "call out" when they wake up rather than manage to soothe themselves back to sleep. It is that ability which treatment needs to develop, and explains the long term treatment failure of medication in such problems.

Klaus Minde, $\mathrm{MD}$ The Montreal Children's Hospital Montreal, Quebec, Canada

1 Kopferschmitt J, Meyer P, Jaege A, et al. Sleep disorders and use of psychotropic drugs in 6-year-old children. Rev Epidemiol Sante Publique 1992;40:467-71. French.

2 Mindell JA. Empirically supported treatments in pediatric psychology: bedtime refusal and night wakings in young children.J Pediatr Psychol 1999;24:465-81.

3 Minde K, Popiel K, Leos N, et al. The evaluation and treatment of sleep disturbances in young children. $J$ Child Psychol Psychiatry 1993;34:521-33. 